\title{
Als Pilger auf dem heiligen Berg Athos
}

\author{
J.-P. Jorisch
}

Kritische Kontrollblicke der Hafenpolizei vergewissern, dass nur Männer die alte Fähre in Ouranopolis (die «Himmelsstadt») besteigen. Beim Auslaufen aus dem Hafen verlässt man die weltliche Kultur. Keine einzige Frau, nicht einmal weibliche Haustiere haben Zugang zu dieser religiösen Männerwelt. Frei von weiblicher Präsenz ist der Athos allerdings nicht, mehrere Klöster verwahren die Knochen weiblicher Märtyrerinnen als Reliquien, und Mönche, welche Ikonen malen, dürfen als einzige ausnahmsweise Hühner halten, da sie für ihre Arbeit frisches Eidotter benötigen.

Auf der 2stündigen Überfahrt nähert man sich in gemächlichem Tempo der Halbinsel Athos mit der über 1000jährigen Mönchsrepublik. Die $50 \mathrm{~km}$ lange und bis $10 \mathrm{~km}$ breite Halbinsel, an deren Ende der über 2000 m hohe Berg Athos thront, ist von üppigem Grün überwachsen, zeigt grösstenteils keinerlei Zivilisationsspuren und hebt sich so von der kargen griechischen Inselwelt $\mathrm{ab}$. Bereits nach einer Stunde Schiffahrt sieht man die ersten Klöster, welche wie mittelalterliche Burgen mit hohen senkrechten Mauern und gitterbewehrten Fenstern und farbigen Kuppeldächern auf den Felsen hoch über der wilden Meeresküste thronen.

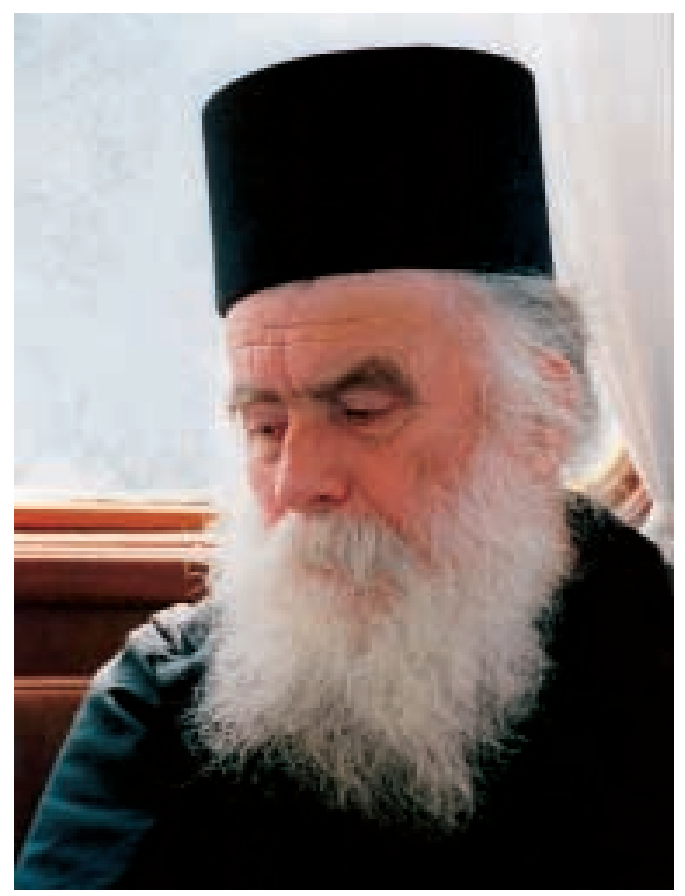

Nirgends auf der Welt besteht eine so grosse Klosterdichte und werden seit so langer Zeit so viele Gebete in den Himmel geschickt. Dieser Umstand lässt den Athos in einem speziellen Licht erscheinen und erklärt seine spirituelle Anziehungskraft.

Von früher 7000 leben heutzutage nur noch 2000 Mönche in 20 Klöstern. Mit weiteren Ablegern und Einsiedeleien sind sie in einer selbstverwalteten «Heiligen Gemeinschaft» organisiert. Ein Parlament von Delegierten aller Klöster regelt das Zusammenleben der Mönche.

Nur zehn ausländische Besucher pro Tag werden zugelassen. Der Aufenthalt ist auf vier Tage beschränkt. Bereits einige Monate vor dem geplanten Besuch muss man sich um eine Einreisegenehmigung bemühen.

Zahlreiche Rekruten verweilen im Rahmen ihrer militärischen Ausbildung einige Tage auf dem Athos. Dies ist ein Hinweis auf die Verankerung des Glaubens in der Bevölkerung, bei uns wäre dies kaum vorstellbar.

Als echter Pilger wandert man von Kloster zu Kloster. Im unwegsamen Gelände sind dies mehrstündige Märsche. Meist sind die Klöster aber durch kleinere Schiffe vom Meer her oder durch Geländefahrzeuge über Staubstrassen ebenfalls erreichbar.

Ausgangspunkt eines Aufenthaltes ist meistens der Verwaltungs- und Hauptort Karies. Er liegt in der Mitte der Halbinsel in einem weiten Bergtal. Ein uralter Klapperbus, der sich in abenteuerlichen Spitzkehren auf einem Schotterweg vom Hafen Dafni zum Dorf hinaufwindet, ist die einzige regelmässige Busverbindung auf Athos. In Karies findet man das einzige Hotel und Restaurant auf Athos, beide von aussen nicht sehr einladend. Einige Läden verkaufen aus staubigen Gestellen Gebrauchsartikel und Lebensmittel für die Klöster und Besucher.

Auf einsamen uralten Wegen wandert man von Kloster zu Kloster. Teils mit schweren Steinen, von Mönchen in Fronarbeit gepflästert, teils auf federndem Waldboden schlängeln sich die Pfade durch den dornigen Urwald. Auf den Feldern arbeitende Mönche bieten frischgepflücktes Obst als Zwischenverpflegung an.

Wenn man sich den Klöstern nähert, ist man immer wieder überrascht von der einzigartigen Architektur. Gegen aussen umgeben hohe Mauern 


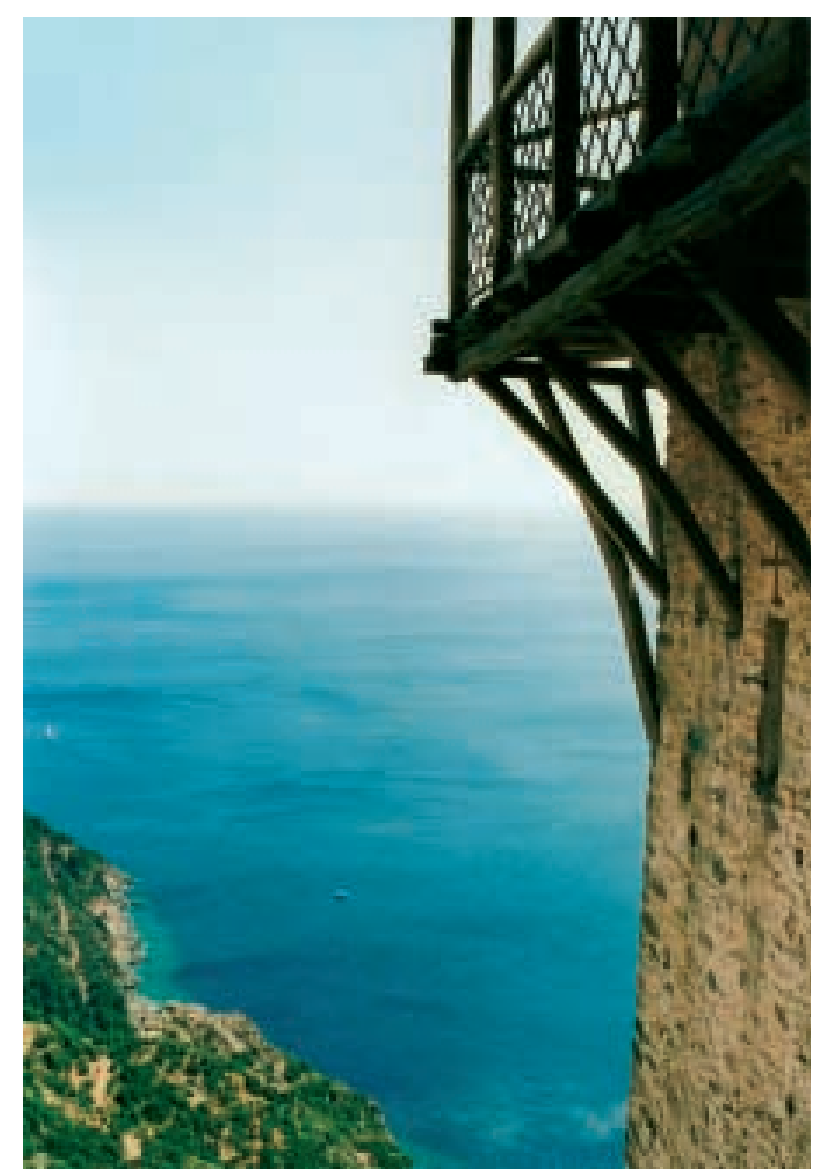

das Kloster, früher ein Schutz gegen Seeräuber und Verbrecher. Nach Einlass durch das tagsüber geöffnete Tor erblickt man innen vielfältige aus den verschiedensten Epochen stammende Kapellen und Gebäude. In den Gängen hängt Petrolgeruch, da nach Anbruch der Dunkelheit mit Petrollampen für Licht gesorgt wird. Geheizt wird im Winter mit Holzöfen, ein schwieriges Unternehmen mit den schlecht schliessenden Fenstern und den meterdicken Natursteinwänden. Elektrizität haben nur wenige Klöster.

Die Klöster sind wahre Museen byzantinischer Kultur. Die Kirchenwände sind mit 800jährigen Fresken und Mosaiken bedeckt, die Altäre und Ikonen mit unvorstellbaren Goldmengen verziert und die Bibliotheken mit unzähligen jahrhundertealten Manuskripten gefüllt. Waghalsig über dem Abgrund abgestützte Balkone mit Ausblick ins unendliche Meer laden zum Nachdenken ein.

Mit einem erfrischenden Glas kaltem Wasser, manchmal einem Kaffee und einer Süssigkeit wird man nach Vorweisung seines «Diamoni tirion», der offiziellen Aufenthaltsbewilligung, vom zuständigen Mönch empfangen. Dann wird man in die spartanische, aber immer saubere Unterkunft geführt. Für Fremde, die nicht dem orthodoxen Glauben angehören, sind es meist etwas abseits vom Kloster gelegene Zimmer.

Die Mönche, zu erkennen an ihren wallen-

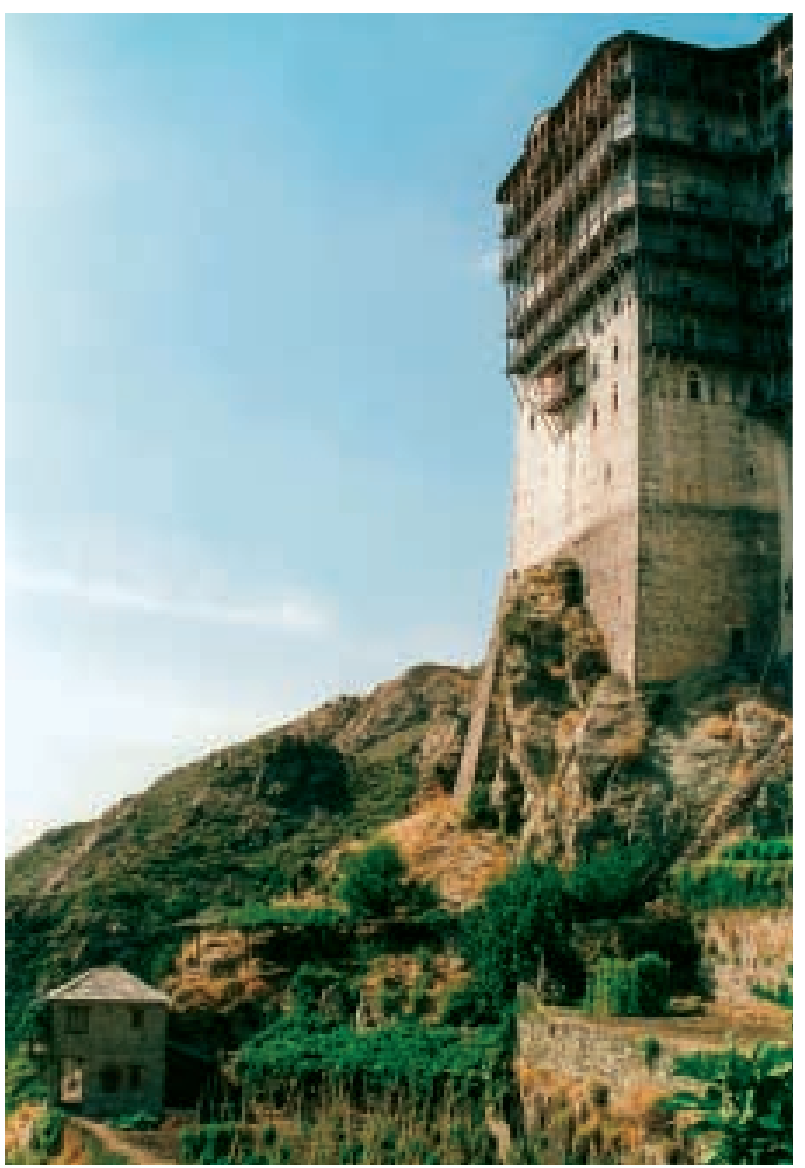
den schwarzen Gewändern, Vollbärten und langen Haaren, die in einem Rossschwanz unter einer Kopfbedeckung stecken, stammen im wesentlichen aus Griechenland. Dennoch trifft man Angehörige vieler ethnischer Gruppen. Sie müssen aber inzwischen die griechische Staatsbürgerschaft annehmen. Auffallend viele sind jung.

Als Nichtorthodoxer erfährt man in den Gesprächen immer noch Spuren der Verletzungen, angehäuft, seit sich die orthodoxe Kirche um die Jahrtausendwende vom römischen Christentum trennte. So bleibt der Zugang zum Allerheiligsten in den Klosterkirchen den Nichtorthodoxen verwehrt. Dennoch wird man Zeuge der devoten Frömmigkeit: Kniefälle, unzählige Kreuzzeichen und Küsse der Heiligenbilder während der stundenlangen Gottesdienste mit abwechselndem Gesang und monotonen Lesungen, Kerzenanzünden und Löschen, Weihrauch und Gebet.

Kleine persönliche anekdotische Begegnungen mit den uniform gekleideten Mönchen lassen einen bald den einen oder anderen wiedererkennen. Ein alter Mönch verteilt während des Gottesdienstes Bonbons an die Besucher.

Dass man als Gast am Rhythmus der Klostergemeinschaft teilnimmt, ist selbstverständlich. 


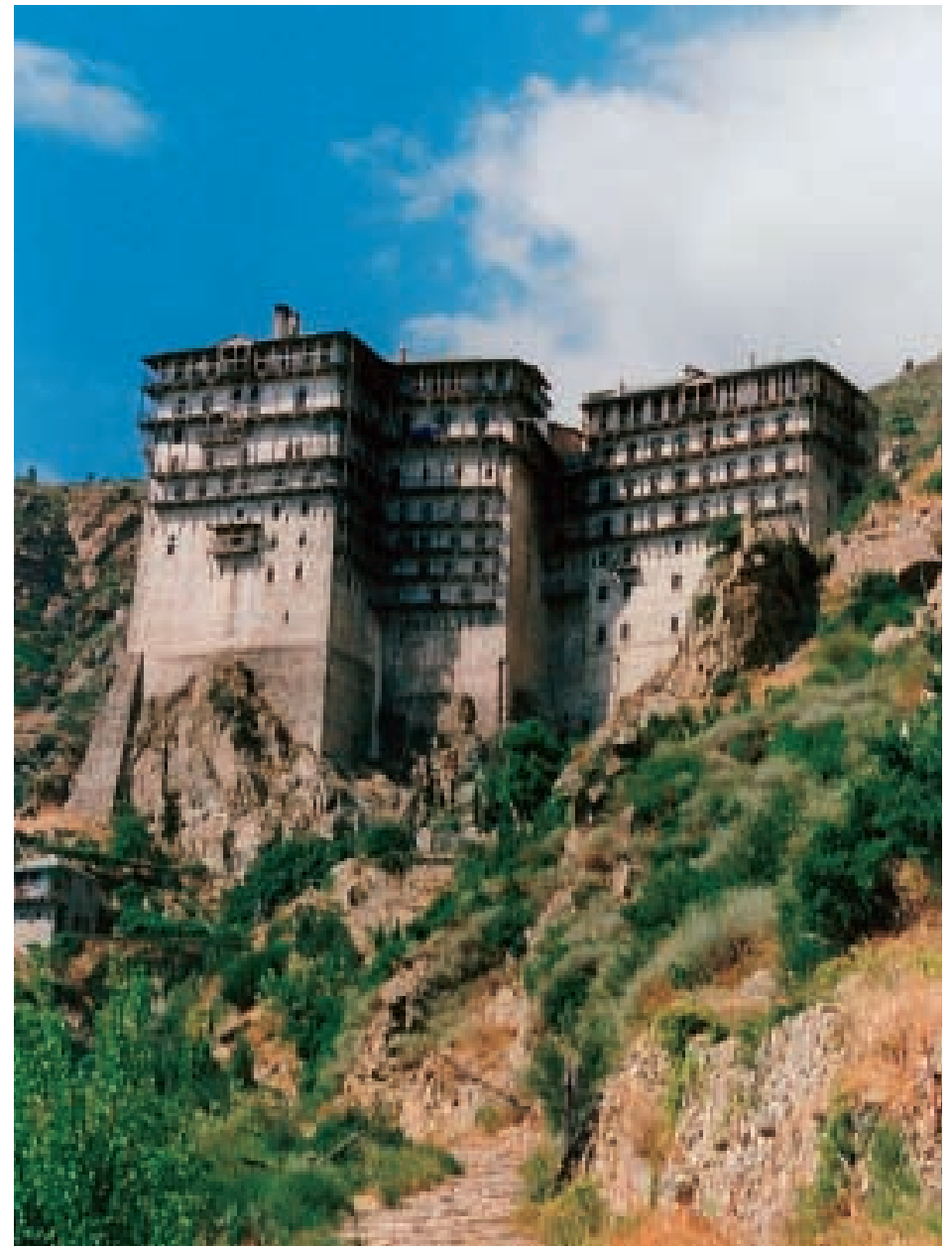

Das Simandron, eine Stundentrommel aus Zypressenholz, ruft mit überlieferten auf- und abschwellenden Rhythmen zum Gebet und bestimmt den Tagesablauf. Mit dieser Trommel soll Noah vor der Sintflut die Tiere in seine Arche gerufen haben. Zwei Zeiten stehen zur Auswahl: die weltliche Zeit und der 24-Stunden-Tag nach dem Sonnenstand, der den Klosterrhythmus bestimmt.

Es wird nach dem alten Julianischen Kalender gelebt, somit sind alle Feiertage um zwei Wochen verschoben.

Mit dem Frühgebet um 4 Uhr morgens beginnt der Tag und endet bei Sonnenuntergang, wenn die Tore des Klosters geschlossen werden, ungeachtet, ob man sich als Gast inner- oder ausserhalb der Klostermauern befindet.

Das Essen ist asketisch, und in Blechgeschirr serviert, dient es zur Lebenserhaltung. Während der gemeinsamen Mahlzeiten wird aus der Bibel vorgelesen. Das Ende des Bibeltextes signalisiert ein abruptes Ende der Mahlzeit. Ob man schon selber fertiggegessen hat oder nicht, spielt keine Rolle.
Mittwoch und Freitag sind Fastentage, an denen die Mönche nur eine einzige dürftige Mahlzeit zu sich nehmen.

Die Regeln schreiben vor, dass Besucher normalerweise jede Nacht in einem anderen Kloster verbringen müssen. Dies erschwert die Pflege von Gesprächen mit den Mönchen. Man hat aber auch den Eindruck, dass die Mönche irgendwie durch das Kommen und Gehen von immer neuen Fremden Abnutzungserscheinungen zeigen.

Auffallend ist die in den meisten Klöstern rege Renovationstätigkeit. Mit Eigenmitteln der wohlhabenderen Klöster, Spenden und dank grosszügiger Unterstützungsgelder der Europäischen Gemeinschaft werden Klostermauern, welche zum Teil ins Meer abzurutschen drohen, aufwendig unterfangen und gestützt. Aber auch im Klosterinnern werden die zerfallenen Gebäude und Kapellen in mühsamer Handarbeit liebevoll wiederaufgebaut und die Klosterinfrastruktur verbessert. Immer mehr neue junge Mönche brauchen ein neues Zuhause.

Der Alltag der Mönche ist streng geregelt und übt vielleicht deswegen die Faszination für die jungen Gläubigen aus. Zwischen den beiden Gottesdiensten am frühen Morgen und am späten Nachmittag gehen sie den verschiedensten Tätigkeiten nach. Einige arbeiten auf dem Felde oder sind sonst handwerklich im Klosterareal tätig. Diese sind meist schon am Körperbau und an den groben Kleidern und Schuhen zu erkennen. Zum Teil werden noch Jeans unter der Kutte getragen. Andere feiner gekleidete und körperlich nicht von Arbeit Gezeichnete verrichten hauptsächlich Denkarbeit in Form von Schriftenstudium, Schreiben und Malerei. Eine grosse Anzahl weltlicher Angestellter verrichtet Hilfsarbeiten, ohne die der Klosteralltag unvorstellbar wäre.

Die einzelnen Klöster zeigen grosse Unterschiede im Reichtum. In einem Kloster werden Medikamentenspenden für erkrankte Mönche erbeten, im anderen Kloster steht neben modernstem landwirtschaftlichem Fuhrpark ein komfortabler Geländewagen modernster Bauart im Innenhof.

Die grosse Zahl der Klöster und die kurze Aufenthaltsdauer erlauben jedoch, nur eine sehr kleine Auswahl zu besuchen. Jedes Kloster hat seine eigene Geschichte und Architektur, eingebettet in die vielfältige Landschaft.

Als Studienreise und Gelegenheit zum Einblick in die griechisch-orthodoxe Religionskultur ist ein Besuch auf Athos eine einmalige Gelegenheit, für spirituelle Erfahrungen ist diese Art der Pilgerreise allerdings nicht geeignet. 